\title{
FURTHER OBSERVATIONS OF THE ACID CONTROL OF THE PYLORUS IN INFANTS *
}

\author{
DAVID MURRAY COWIE, M.D., AND WILLIAM LYON, M.D. \\ ANN ARBOR
}

In a former paper ${ }^{\mathbf{1}}$ we recorded two experiments on the acid control of the pylorus in infants. We have since extended and verified these results and present them herewith, together with a partial description of the first experiments.

\section{HISTORICAL NOTE}

The explanation of the opening and closing of the pylorus was not fully made until Pawlow and Cannon's work was done. Not long after Beaumont's experiments in 1832 it is true that Hirsch, by means of a duodenal fistula in a dog, came to the conclusion that acid on the duodenal side caused the pylorus to close, but he abandoned his idea in favor of von Mering's observations, made about the same time, that the closure of the pylorus was due to the bulk of liquid or food in the duodenum. In his experiments von Mering introduces milk into the duodenum. The observation that the mechanical effect of inflating a small rubber balloon in the duodenum causes the pylorus to contract and its rhythmic movements to cease, seemed to lend support to von Mering's theory.

It was left for Pawlow and Cannon, however, to clear up these discrepancies and make full explanation of the phenomena observed by Hirsch and von Mering. Pawlow demonstrated that milk or neutral fat in the duodenum, whether in small or large bulk, excites the duodenal closing reflex and keeps the pylorus closed until the neutral fat has become saponified or until it has passed on into a lower segment of the intestine. He also showed that acid on the duodenal side closed the pylorus. Later Cannon demonstrated that acid in the stomach opens the pylorus and that the differential escape of carbohydrates and proteins from the stomach is due to their respective stimulating and combining property for hydrochloric acid. Hence the observations of Hirsch and von Mering were correct, but their explanations were entirely faulty.

\section{AUTHORS' EXPERIMENTS}

The experiments which resulted in the above observations were performed on dogs and cats. Of the various methods used Cannon's is

*From the Pediatric Clinic, University of Michigan.

* Read at the Meeting of the American Pediatric Society, Lake Mohonk, May, 1911.

1. Arch Pediat., February, 1911. 
probably ideal, as it removed practically all abnormal conditions. In conducting our experiments we have been aware of the arguments against the accuracy of the method of tubing the stomach and we have not lost sight of the possible effect of the preantral sphincter on our results. The small size and the elasticity of the infant's stomach better adapt it for such observations than the adult stomach, and for these reasons we believe that the method we used, properly controlled, fully demonstrates the point at issue.

Method.-We selected babies who had proved their indifference to the passage of the tube. That is, they had become accustomed to the tube and retained it with perfect ease for any period desired. They would often sleep throughout an experiment, excepting for the brief interval of passing the tube. We found No. 14 (English) soft rubber catheter connected by a piece of glass tubing with a rubber tube and small bulb the most satisfactory apparatus for our work. As we had not investigated the effect of posture on the rate of stomach evacuation we had the position of the infant constant; that is, lying on the left side.

Control Test Meal.-Skim-milk, diluted one-half with water, was employed as a suitable test meal for these investigations. The meal usually contained a fraction of 1 per cent. of fat and proved more convenient to work with than milk containing a higher fat per cent., and this removed from discussion the possible effect of fat on the pyloric reflex. A definite amount was delivered to the stomach through a tube and allowed to remain a definite time. It was then recovered by expression and syphonage as described below.

Acid Meal.-This meal was prepared by treating skim-milk with dilute hydrochloric acid (10 per cent. solution) until the acidity desired was obtained. The food was then kept at body temperature for an hour to insure complete combination of the acid with the protein. It was then retested and treated, if necessary, to insure the acid value as it entered the stomach.

Basic Meal.-The basic calcium casein meal was prepared by adding the requisite amount of lime water to the skim-milk to render it faintly but distinctly alkaline to phenophthalein. The balance of the dilution was then made up with water so that, as with the control and acid meal, the skim-milk formed half of the mixture. This meal was also retested to insure its alkalinity as it entered the stomach.

Calculation of Error.-It could not be expected that work of this kind would be trustworthy for comparisons, excepting in marked differences. To ascertain what our error would be in infants who had not been previously tubed or selected for investigation because of their particular adaptability, we made sixteen observations on infants taken at random. These are recorded in Table 1 . In most of these cases the infants were 
allowed to go three and a half hours after a feeding before giving the test-water. If the stomach was empty we introduced 45 c.c. of plain water through the catheter, recovering it immediately by expression and syphonage. In each case the water was completely delivered to the stomach, none being allowed to remain in the tube. The syphonage was started by expression, one of us pressing over the epigastrium. It will be seen that our greatest error in recovering the contents is 5 c.c. and that the error is seldom greater than 3 c.c. In most instances a little mucus was recovered, As stated before, the infants with which this investigation had to do were carefully selected and caution was observed to wash and drain the stomach. In these babies our error ${ }^{1}$ was less than 3 c.c.

Table 1.-Sixteen Investigations on Infants Taken at Random to Deter-

mine Rovghty the Error in Recovering ImMediately by Means of Stomach Tube, a Given Amount of Fluid Delivered to the Stomach by Gavage

\begin{tabular}{|c|c|c|c|c|c|c|}
\hline $\begin{array}{l}\text { No. } \\
\cdots \\
\cdots \\
\ldots\end{array}$ & $\begin{array}{l}\text { Name } \\
\ldots \ldots \\
\ldots \ldots \\
\ldots \ldots\end{array}$ & $\begin{array}{l}\text { Interval } \\
\text { Since Last } \\
\text { Feeding } \\
\text { hours }\end{array}$ & $\begin{array}{c}\text { Condition } \\
\text { of } \\
\text { Stomach } \\
\ldots \ldots\end{array}$ & $\begin{array}{l}\text { Amount } \\
\text { of water } \\
\text { Given, } \\
\text { c.c. }\end{array}$ & $\begin{array}{l}\text { Amount } \\
\text { of water } \\
\text { Recovered, } \\
\text { c.c. }\end{array}$ & $\begin{array}{c}\text { Condition } \\
\text { of water } \\
\text { Recovered } \\
\text {........ }\end{array}$ \\
\hline 1 & Edwards & $\ldots 3^{1 / 2}$ & Empty & 45 & 40 & About $1 / 2$ c.c. mucus \\
\hline 2 & Hessler & $\ldots 31 / 2$ & Empty & 45 & $451 / 2$ & $\ldots \ldots \ldots \ldots \ldots$ \\
\hline 3 & Rush ... & $\ldots 31 / 2$ & Empty & 45 & $46^{2}$ & A little mucus \\
\hline 4 & Kinyon & $\ldots .21 / 2$ & Empty & 45 & 46 & Tinged yellow \\
\hline 5 & Wileox & $\ldots .31 / 2$ & Empty & 45 & 41 & \\
\hline 6 & Rush ... & $\ldots . .31 \%$ & 2 c.c. & 45 & 43 & Few fine curds \\
\hline 7 & Mills ... & $\ldots 3^{1 / 2}$ & Empty & 45 & 40 & A little mucus \\
\hline 8 & Hessler & $\ldots .31 / 2$ & Empty & 45 & $451 / 2$ & Plugs of mucus \\
\hline 9 & Simmons & $\ldots 31 \%$ & Empty & 45 & 46 & Considerable mucus \\
\hline 10 & Hackford & $\ldots 31 / 2$ & Empty & 45 & 49 & 2 or 3 c.c. mucus \\
\hline 11 & Hacket. & $\ldots 31 / 2$ & Empty & 45 & 44 & $1 / 2$ c.c. thick mucus \\
\hline 12 & Rush .. & $\ldots 31 / 2$ & Empty & 45 & 41 & Few fine curds \\
\hline 13 & Hatzke & $\ldots . .31 / 2$ & Empty & 45 & 40 & Little thick mucus \\
\hline 14 & Mills . . & $\ldots . .31 / 2$ & Empty & 45 & 44 & Few fine curds \\
\hline 15 & Nering. & $\ldots .31 / 2$ & Empty & 45 & 45 & A little mucus \\
\hline 16 & Hessler & $\ldots . .31 / 2$ & Empty & 45 & 41 & A little mucus \\
\hline
\end{tabular}

SERIES I.-THE EFFECT OF ACID ON THE DUODENAL SIDE OF THE PYLORUS

The three sets of experiments recorded in Charts 1, 2 and 3 have to do with the duodenal side of the pyloric reflex. The control meals enter the stomach, remain there for a certain time, until the acid reflex on the stomach side causes the pylorus to open. The acid meal enters the stomach with immediate stimulus for the pyloric opening reflex. As soon as it reaches the duodenum, in proportion to the amount ejected and its acidity, the pylorus remains closed. As our acid meal causes the pylorus to remain closed a comparatively long time, we speak of this as a sustained duodenal reflex, in contradistinction to the normal pyloric reflex and the delayed pyloric reflex recorded in our second series.

CASES 1 AND 2.-The data relating to these infants will be found in our prerious report. ${ }^{1}$ Both were in good health and gaining in weight. At no time

2. Greatest error in any one case 5 c.c. Average error 2.38 c.c. 


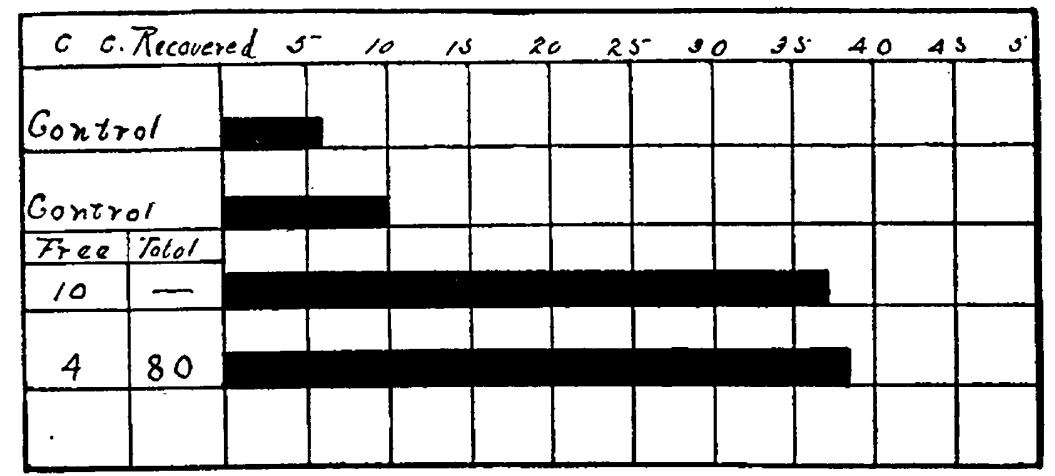

Chart 1.-Sustained duodenal reflex, Case 1.

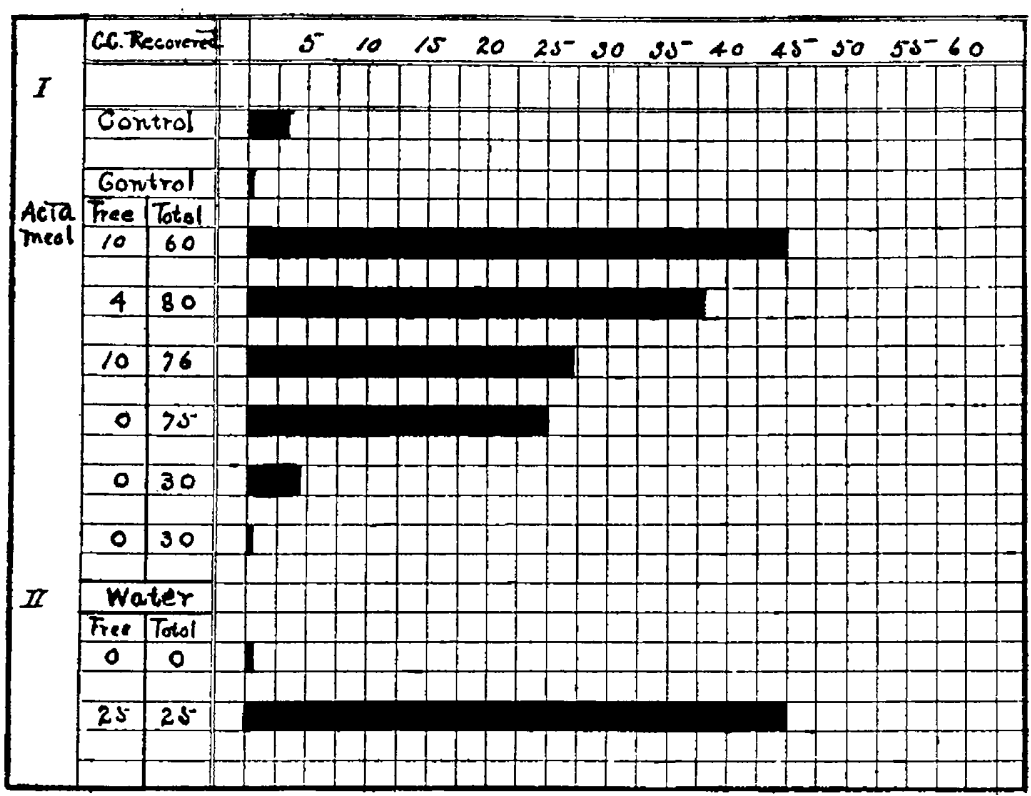

Chart 2.-Sustained duodenal reflex, Case 2. The secretion of this infant's stomach was always normal. There was no free $\mathrm{HCl}$.

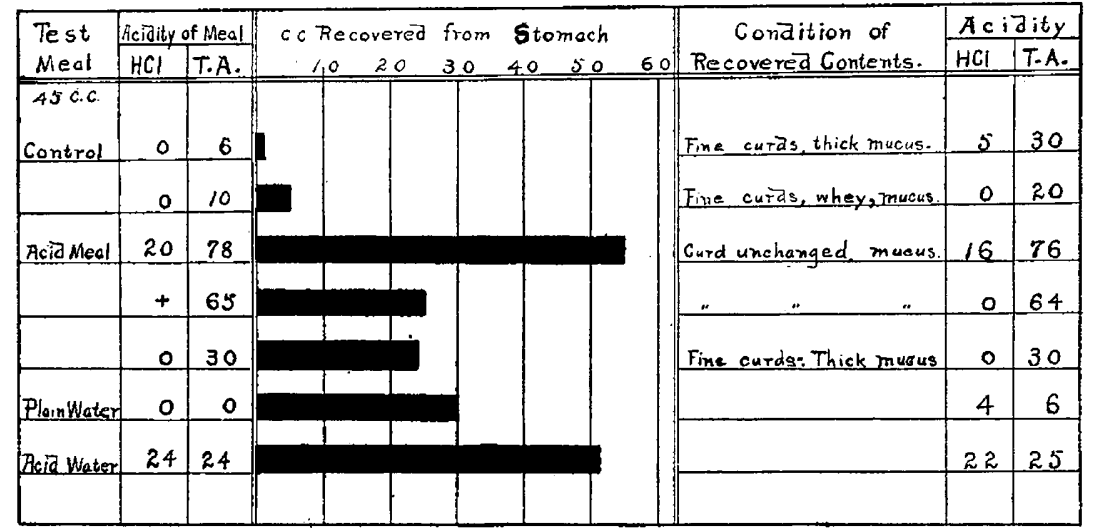

Chart 3.-Sustained duodenal reflex, Case 3. The secretion of this infant's stomach was always hypernormal. Free acid occurred in the fasting stomach. 
in either case were we able to find any free hydrochloric acid. The delayed evacuation of the stomach is well shown in the acid meals. The higher the acidity, or the more acid present, the larger the amount recovered at the end of the period. This is particularly well shown in Case 2.

CASE 3.-A well developed baby. History and physical examination negative. On referring to the series of stomach analyses (Table 2) it will be seen that the infant almost always secreted acid to the free point and at times gave a free acid value as high as 25 and 35 , a true hyperchlorhydria for an infant. In addition to this, there were evidences of hypersecretion during the period of our observations. How long such a condition would continue without producing spasm, if it ever would, we cannot say, as the infant soon passed from under our observation.

There was nothing in Case 3 to suggest ulcer, either peptic or duodenal. We find in this case, three hours after an ordinary feeding, when the stomach is practically free from food remains, a free acid value of 5 . We also find that plain water leaves this baby's stomach much more slowly than does the control milk meal and the water meal in Case 2. It is believed, and supported by experiment, that water leaves the stomach almost directly. The reason for this, however, has never been satisfactorily explained. In this case we think the delayed evacuation of water can be explained by the presence of free acid in the fasting stomach and the hypersecretion. The water does not take up the acid but, as is known, may provoke a further secretion in this neurosis, hence the duodenal reflex is sustained longer than under normal conditions. In Case 2 we had no such condition to contend with. Free acid was never detected and the total acidity after an ordinary or test meal was never high, accordingly there was little or no neutralization to take place in the duodenum and the water passed on uninterruptedly into the gut.

Table 2.-Results of Experimental Feeding in Case 3, Showing Hyper. SECRETION AND HYPERCHLORHYDRIA*

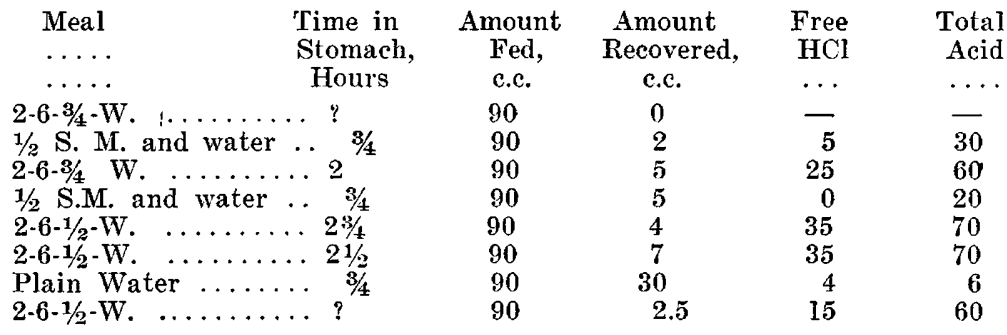

*Abbreviations : $2-6-3 / 4=2 \%$ fat, $6 \%$ sugar, $3 / 4 \%$ protein. $W=$ whey made up the balance of the mixture. $1 / 2$ S.M.=Half skim milk and water.

\section{DETAILED ACCOUNT OF EXPERIMENTS}

Control Meal No. 1. Infant 36 Days Old. May 11, 1910

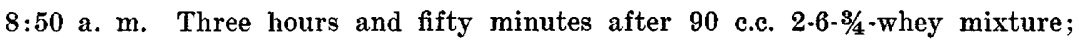
nothing obtained through tube; stomach washed with 90 c.c. water; all recovered, containing about 1 per cent. fine curds and mucus. 
s:55 a. m. Fed 90 c.c. half skim-milk and water, through a No. 14 catheter; total acidity of food; fat .9 per cent.

$9: 40$ a. m. (45 minutes) 2 c.c. whey; mucus thick; a few very fine curds recovered; stomach washed with 85 c.c. water; 83 c.c. containing about 2 per cent. thick mucus and a few small curds recovered. Free $\mathrm{HCl} 5$; total acid 30 .

Control Meal No. 2. Infant 37 Days Old. May 12, 1910

8:40 a. mf. Two hours and forty minutes after 90 c.c. of $2-6-3 / 4$ whey mixture; 5 c.c. recovered, containing 50 per cent. curds, and mucus thick. HCl 25 ; total acid 60 . Stomach washed with 40 c.c. warm water; 40 c.c. recovered practically clear.

$8: 45$ a. m. Five minutes later, fed by gavage 90 c.c. half skim-milk and water; total acid 10 ; fat .8 per cent.

$9: 30$ a. $\mathrm{m}$. (45 minutes) 5 c.c. recovered; whey containing a few small curds and mucus. $\mathrm{HCl} 0$; total acid 20 .

Acid Meal No. 1. Infant 38 Days Old. May 13, 1910

6:00 a. m. 90 c.e. $2-6-1 / 2$ whey nursed.

8:45 a. m. 4 c.c. recovered, containing 25 per cent. thick mucus and a few small curds, HCI 35 ; total acid 70 . Stomach washed with 45 c.c. warm water; all recovered, containing 2 per cent. thick mucus.

$9: 00$ a. m. Fed 90 c.c., half skim-milk and water acidified by gavage. Free $\mathrm{HCl} 20$; total acid 78 .

$9: 45$ a. $\mathrm{m}$. (45 minutes) 55 c.c. milky material recovered containing 20 per cent. very fine curds and thick mucus. The whey is very milky. $\mathrm{HCl} \mathrm{16;} \mathrm{total} \mathrm{acid} 76$. Stomach washed with 45 c.c. warm water; all recovered; slightly opaque with milky material.

Acid Meal No. 2. Infant 42 Days Old. May 16, 1910

$9: 45$ a. m. 90 c.c. half skim-milk and water, acidified, fed. Free HCl trace; total acid 65 .

10:30 a. m. (45 minutes) 25 c.c. recovered. Same condition as when it entered stomach, excepting a little mucus. $\mathrm{HCl} 0$; total acid 64. Stomach washed with 80 c.c. water; 82 c.c. recovered; slightly turbid.

Acid Water Meal Experiment. Infant 42 Days Old. May 16, 1910

6:00 a. m. 90 c.c. $2-6 \cdot 1 / 2$ whey nursed.

8:30 a. m. 7 c.c. recovered containing 50 per cent. thick mucus and curds. $\mathrm{HCl} \mathrm{35}$; total acid 70 . Stomach washed with 45 c.c. warm water; 46 c.c. recovered, slightly cloudy.

$s: 40$ a. m. Fed 90 c.c. plain water by gavage.

$9: 10$ a. m. 30 c.c. recovered containing a little thick mucus. $\mathrm{HCl} \mathrm{4;} \mathrm{total}$ acid 6 .

$9: 13$ a. $\mathrm{m}$. Fed 90 c.c. acidified water, $\mathrm{HCl} 24$, by gavage.

$9: 43$ a. m. (30 minutes) 51 c.c. recovered. Free $\mathrm{HCl} 22$; total acid 25.

\section{Acid Milk Meal No. 3. Infant 43 Days Old. May 1\%, 1910}

8:55 a. m. 2.5 c.c. containing 60 per cent. thick mucus and curds recovered from stomach. Free HCl 15; total acid 60 . Stomach washed with 95 c.c. warm water; 90 c.c. recovered containing 2 per cent. fine curds and mucus.

$9: 00$ a. m. 90 c.c. half skim-milk and water, total acidity 30 , given by gavage.

$9: 45$ a. m. ( 45 minutes) 24 c.c. clear whey containing a few small curds and considerable thick mucus recovered. $\mathrm{HCl} 0$; total acid 30 . Stomach washed with 45 c.c. warm water; all recovered practically clear. 
SERIES II.-THE EFFECT OF ACID ON THE STOMACH SIDE OF THE PYLORUS

In this series the control meal enters the stomach under the same conditions as before; there is a food stimulus for the secretion of acid to open the pylorus, while in the basic or alkaline meal, although the food stimulus, so far as we know, is exerted on the secretory mechanism of the stomach, the presence of alkali to the point of saturation of the protein delays the acid in reaching the point for stimulation of the opening reflex; hence at the end of forty-five minutes a very much larger amount is recovered than in the control experiments-not 5 or 10 c.c., but double and treble the amount.

It will be observed in these experiments that at times we recovered as much as 15 c.c. more, at the end of the period, than we had put in. This was at a time preceding the opening reflex and represents roughly the amount of gastric juice secreted. In Case 4 we recovered at the end of thirty minutes 15 c.c. more than we put in; at the end of forty-five minutes we recovered a few c.c. more than we originally put in, but about 12 c.c. less than we reintroduced into the stomach. In this experiment, if we had again introduced the contents removed at forty-five minutes, ten minutes later we might have experienced what we have on more than one occasion-the stomach almost empty. The pyloric reflex has just begun to operate and because of the low acidity of the food either a large gush or a series of rapid short gushes has evacuated the stomach. On one occasion at forty-five minutes nearly all the food was present in the stomach, and no curd formation had taken place; in fifty minutes only a small amount of curded material could be recovered.

These experiments, we think, give abundant proof that the pylorus is governed by acid on both sides of the pyloric ring and confirm, in man, the experiments in animals done by Pawlow, Cannon and others.

CAse 4 (Charts 4 and 5).-Baby G., an apparently normal infant. History and physical examination negative. On referring to the series of stomach analyses, Table 3 , it will be seen that this infant never secreted free acid and that the total acid was never excessively high.

Table 3.-Results in Free HCl and Total acidity in Feeding Experiments IN CASE 4

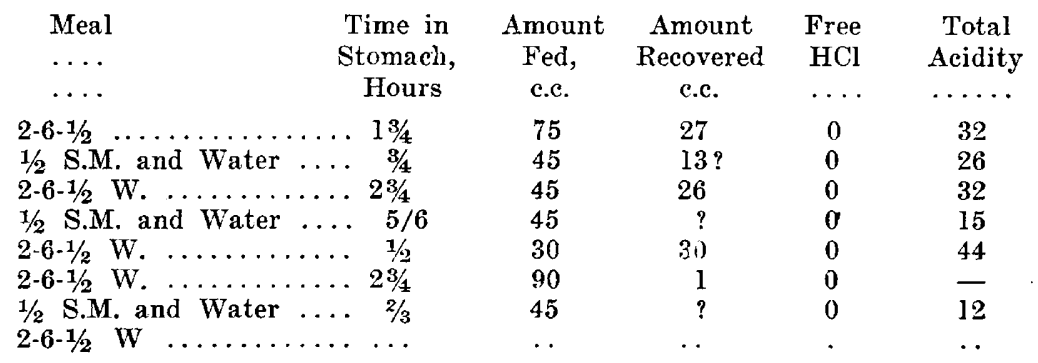


DETAILED ACCOUNT OF EXPERIMENTS IN CASE 4

Control Meal No. 1. Infant 30 Days Old. May 25, 1910

$7: 30$ a. $\mathrm{m}$. Nursed 75 c.c. $2-61 / 2$ mixture.

$9: 13$ a. m. 27 c.c. recovered, containing 15 per cent. curds and thick mucus. $\mathrm{HCl} 0$; total acid 32 . Stomach washed with 90 c.e. water; 90 c.c. recovered, containing 2 per cent. curds.

$9: 20$ a. m. 45 c.c. half skim-milk and water, total acidity 10 , given by gavage. $9: 50$ a. m. ( 30 minutes) 13 c.c. of fluid were expressed, consisting of whey, medium sized curds, and thick mucus. This was returned to the stomach.

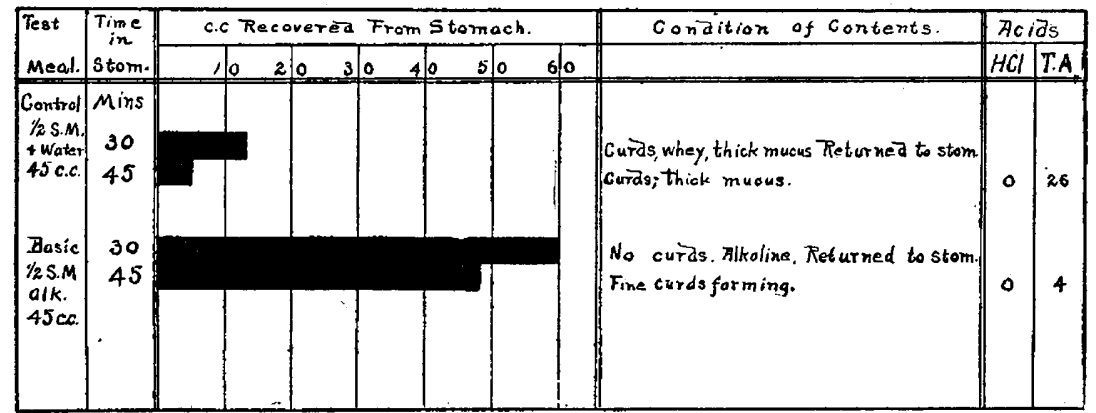

Chart 4.-Delayed pyloric reflex. Case 4, Experiment 1, showing the effect of alkali on stomach evacuation. The secretion of this infant's stomaoh was always normal.

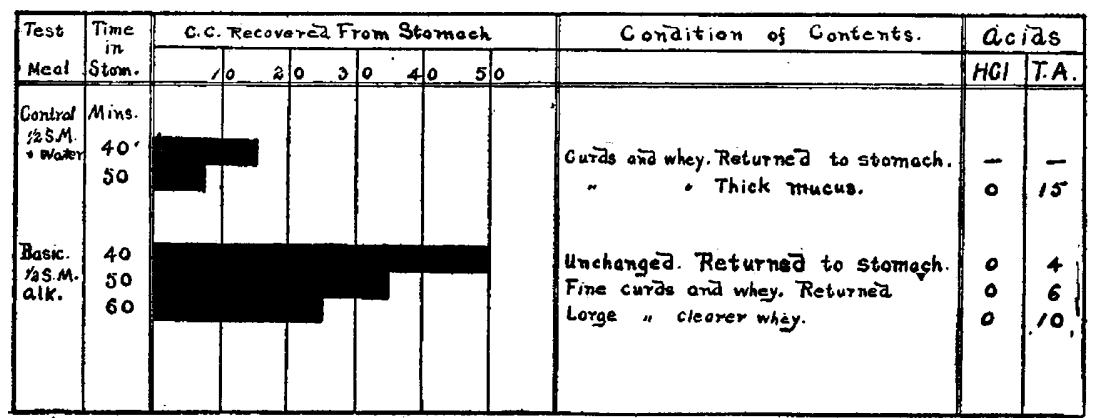

Chart 5.-Delayed pyloric reflex. Case 4, Experiment 2, showing the effect of alkaline contents on stomach evacuation.

10:05 a. m. (45 minutes) 15 c.c. of fluid were expressed consisting of 20 per cent. large curds and some thick mucus. $\mathrm{HCl} 0$; total acid 26. Stomach washed with $90^{\circ}$ c.c. water; 88 c.c. returned practically clear.

Basic Meal No. 1. Infant 30 Days Old. May 25, 1910

10:10 a. m. 45 c.c. of half skim-milk and water, rendered basic with lime water, were given by gavage.

$10: 40$ a. m. (30 minutes) 60 c.c. recovered; no curds; reaction alkaline to litmus; returned to stomach.

10:55 a. m. (45 minutes) 49 c.c. Fine curds beginning to form. $\mathrm{HCl} 0$; total acid 4. Stomach washed; only a few fine curds returned. 
Remark: It will be observed that thirty minutes after feeding we recovered 15 c.c. more than was introduced, and that no curd formation was present; that fifteen minutes later curds had formed and 45 c.c. were recovered. No stimulus to pyloric opening was present until about this time. The 15 c.c. doubtless represents grossly the amount of gastric juice secreted during the first thirty minutes' stay in the stomach. See Chart 4.

\section{Control Meal No. 2. Infant 32 Days Old. May 27, 1910}

6:00 a. m. Fed 45 c.c. $2-6-1 / 2$ whey mixture.

$8: 40$ a. $m$. 26 c.c. recovered; 30 per cent. curds and thick mucus. $\mathrm{HCl} \mathrm{0}$; total acid 32. Stomach washed with 90 c.c. warm water; all recovered, containing a little over 1 per cent. fine curds and mucus.

$8: 45$ a. m. 45 c.c. half skim-milk and water, total acid 9 , given by gavage.

$9: 25$ a. m. ( 40 minutes) 15 c.c. expressed, consisting of fine curds and whey. Returned to stomach.

$9: 35$ a. m. (50 minutes) 8 c.c. fluid containing 20 per cent. medium small curds and a little thick mucus recovered. HCl 0 ; total acid 15. Stomach washed with 90 c.c. water; 89 c.c. recovered, containing a few fine curds and mucus hardly measurable by centrifuge.

Basic Meal No. 2. Infant 32 Days Old. May 27, 1910

$9: 40$ a. m. 45 c.c. one-half skim-milk and water, rendered basic with lime water, were given by gavage.

10:20 a. m. (40 minutes later) 50 c.c. recovered; no distinct curds; appears about the same as when introduced. Total acid 4; returned to stomach.

10:30 a. m. (50 minutes) 35 c.c. recovered; distinct fine curds and whey. Free acid 0 ; total acid 6 . Returned to stomach.

10:40 a. m. (60 minutes) 25 c.c. recovered; curds larger and more separated from the whey. $\mathrm{HCl} 0$; total acid 10. Stomach washed clear.

Basic Meal No. 3. Infant 36 Days Old. May 31, 1910

$5: 00$ a. $m .75$ c.c. $2-6-1 / 2$ whey mixture.

$8: 00$ a. $\mathrm{m}$. 15 c.c. $2-6-1 / 2$ whey mixture.

$8: 30$ a. m. 30 c.c. of whey containing 2 per cent. curds and a little thick mucus recovered. HCl 0; total acid 44. Stomach washed with 130 c.c. warm water. All recovered plus a little mucus.

8:55 a. m. 45 c.c. one-half skim-milk and water, rendered basic, given by gavage.

$9: 35$ a. m. (40 minutes) 35 c.c. recovered, containing 20 per cent. curds, whey and thick mucus. Tube was repeatedly blocked by the curds. HCI 0 ; total acid 8 . Stomach washed with 90 c.c. warm water; 90 c.c. recovered, containing a few small curds and considerable thick mucus.

Control Meal No. 3. Infant 38 Days Old. June 2, 1910

$6: 00$ a. m. 90 c.c. $2-6-1 / 2$ whey mixture.

$8: 45$ a. $\mathrm{m}$. 1 c.c. thick mucus recovered. $\mathrm{HCl} 0$. Stomach washed with 45 c.c. warm water; all recovered, perfectly clear excepting a little mucus.

8:55 a. m. 45 c.c. plain one-half skim-milk and water were given by gavage; total acidity 8.

$9: 15$ a. m. ( 20 minutes) 45 c.c. recovered; fine curds beginning to form. Returned to stomach.

9:25 a. m. (40 minutes) 30 c.c. recovered; curds more distinct; mucus more marked. Returned to stomach.

$9: 35$ a. $\mathrm{m}$. (50 minutes) 15 c.c. recovered, whey containing 5 per cent. large curds; tube blocked with thick mucus. $\mathrm{HCl} 0$; total acid 12. Stomach washed clean. 
It may be asked what practical bearing these observations have on gastro-enterologic therapy and on our knowledge of gastro-enteric diseases. It was Dr. Cowie's object in starting these observations to see if the management of pyloric stenosis in infants, both true and false, could be put on a scientific basis. Most cases of this character have been treated without reference to the pyloric reflex or the altered physiologic conditions present. We believe there is a certain group of cases in which these are important factors to determine. It would seem at least possible to recognize varying degrees of stenosis and readily to differentiate them from what may be called pseudo-stenosis or spasm and to enable us to observe the effect of the reaction of the food given on the duration of the duodenal closing reflex, which seems to be an important factor in many cases. This will be considered in a later article.

\section{CONCLUSIONS}

1. The pyloric opening and closing reflex can be easily demonstrated in infants' stomachs.

2. In experimental acid stomach contents the duodenal closing reflex is sustained and consequently the evacuation of the stomach is delayed.

3. In experimental alkaline stomach contents the pyloric opening reflex is delayed and consequently the evacuation of the stomach is delayed.

4. Free acid is not necessary for pyloric opening. Its presence has a tendency to provoke prolonged duodenal closing in an infant.

5. In hypersecretion in infants protein-containing food appears to leave the stomach more readily than water because of its binding property for acid.

Lawrence Building. 